\title{
Targeted electrode-based modulation of neural circuits for depression
}

\author{
Helen S. Mayberg \\ Department of Psychiatry and Department of Neurology, Emory University School of Medicine, Atlanta, Georgia, USA.
}

\begin{abstract}
During the last 20 years of neuroscience research, we have witnessed a fundamental shift in the conceptualization of psychiatric disorders, with the dominant psychological and neurochemical theories of the past now complemented by a growing emphasis on developmental, genetic, molecular, and brain circuit models. Facilitating this evolving paradigm shift has been the growing contribution of functional neuroimaging, which provides a versatile platform to characterize brain circuit dysfunction underlying specific syndromes as well as changes associated with their successful treatment. Discussed here are converging imaging findings that established a rationale for testing a targeted neuromodulation strategy, deep brain stimulation, for treatment-resistant major depression.
\end{abstract}

Depression affects at least $10 \%$ of the world population and is a leading cause of worldwide disability (1). Major depressive disorder (MDD) is clinically defined as a multidimensional syndrome, involving disruption of mood, cognition, sensorimotor functions, and homeostatic/drive functions (including those that control sleep, appetite, and libido). While depression can be treated in many cases with either medication or an evidence-based psychotherapy, remission rates in controlled trials using currently available treatments rarely exceed $30 \%$, and relapse is the rule rather than the exception (2). For many patients, combinations of multiple medications and electroconvulsive therapy (ECT) are required. For those who remain severely depressed despite these aggressive approaches, new strategies are needed (3). This Review describes the development and testing of a new interventional strategy, deep brain stimulation (DBS), directed at this group of patients who are otherwise resistant to treatment.

Critical to the development of DBS as a new treatment for intractable MDD has been the evolving understanding of the brain circuits that mediate normal and abnormal mood states and the systematic characterization of changes in these circuits that accompany successful and unsuccessful response to various treatments, measured using functional imaging (reviewed in refs. 4-8). Based on brain circuit models of depression derived primarily from PET scan measures of glucose metabolism and blood flow, the first region of the brain to be targeted with DBS in patients with treatment-resistant depression (TRD) was the subcallosal cingulate (SCC), the ventral-most segment of the cingulate gyrus (Figure 1). As detailed in the following sections, this region of the brain was considered a critical node within a putative mood regulatory circuit, as it showed the most consistent changes in metabolism and blood flow with clinical recovery, using various well-established antidepressant interventions (9). As such, it was hypothesized that direct stimulation of the SCC and adjacent

Conflict of interest: The author consults with Advanced Neuromodulation Systems (ANS, a division of St. Jude Medical [SJM]) and has intellectual property rights on a deep brain stimulation technology for treating depression that has been licensed to ANS/SJM.

Nonstandard abbreviations used: BA, Brodmann area; CBT, cognitive behavioral therapy; DBS, deep brain stimulation; ECT, electroconvulsive therapy; $\mathrm{AMRI}$, functional MRI; MDD, major depressive disorder; PD, Parkinson disease; SCC, subcallosal cingulate; TRD, treatment-resistant depression.

Citation for this article: J. Clin. Invest. 119:717-725 (2009). doi:10.1172/JCI38454. white matter would produce modulatory or normalizing changes within this otherwise unresponsive mood circuit, resulting in antidepressant effects (10). The first clinical results of DBS of the SCC were encouraging, leading to the initiation of additional clinical studies $(11,12)$. This initial clinical success further motivated continued refinement of this depression circuit model, with goals to optimize surgical targeting, define biomarkers that might effectively guide patient selection, and increase understanding of DBS mechanisms of action.

\section{Neurological depression circuit model: theoretical framework}

The development and evolution of a circuit model to explain the many regional brain abnormalities reported in numerous imaging studies of depressed patients is in keeping with the classical neurological tradition of symptom localization, using lesion-deficit correlational analyses. Using this strategy, construction of a depression circuit first assumes that depression is unlikely to be the result of injury or dysfunction of a single brain region but rather a system-wide disorder, in which interruption at specific sites or "nodes" within a defined functional circuit or network linking many brain regions can result in stereotypic depressive symptoms $(9,13)$. It is further hypothesized that a major depressive episode reflects the failure to appropriately regulate activity in this multiregion circuit, under circumstances of cognitive, emotional, or somatic stress (6). From this perspective, a given scan pattern is a readout of both the inciting insult or provocation and the resulting compensatory processes, influenced by various contributing factors, including heredity, temperament, early-life experiences, and previous depressive episodes (14-17). As such, variations in brain scan patterns among patients would be expected, thus defining distinct depression phenotypes with potentially predictable responses to different antidepressant treatments (Figure 2). While this premise is purely speculative, published findings that support this hypothesis are reviewed below, highlighting disease-, state-, and treatment-specific effects facilitated by pharmacotherapy, cognitive behavioral therapy (CBT), and DBS.

\section{Defining affected brain regions}

Structural imaging studies. Structure-function correlations performed in patients developing a depression syndrome, in the context of either acquired brain lesions $(18,19)$ or neurodegenerative disorders 


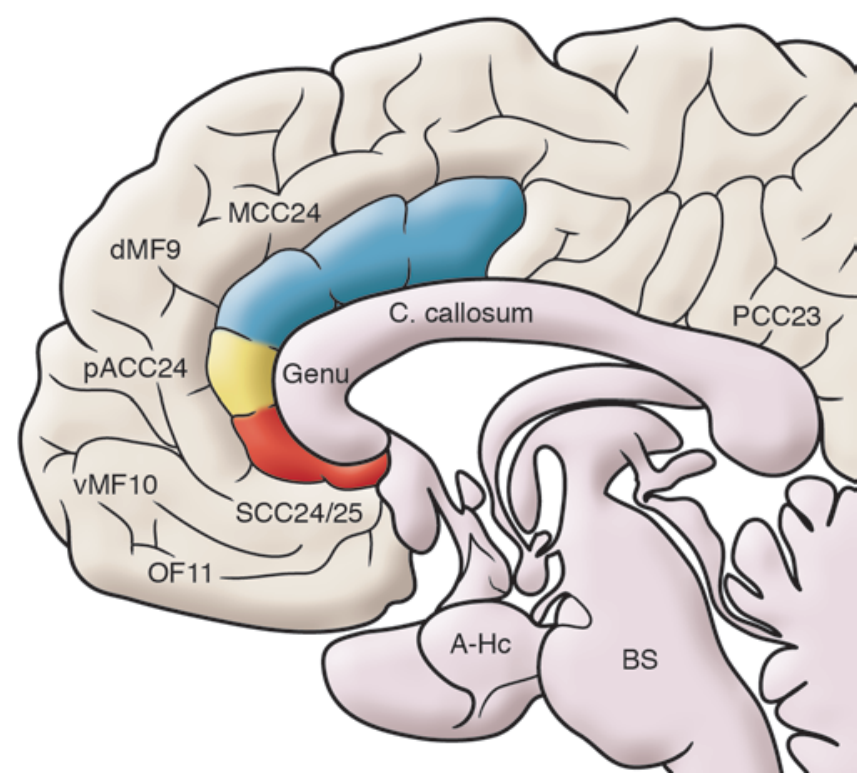

Figure 1

Anatomical locations of key brain regions implicated in MDD. Midline, sagittal view of the brain, with subsections of the anterior cingulate cortex highlighted by coloring. A-Hc, amygdala hippocampus; BS, brainstem; C. callosum, corpus callosum; dMF9, dorso-medial frontal cortex BA9; MCC24, mid-cingulate cortex BA24 (blue); OF11, orbital frontal cortex BA11; pACC24, pregenual anterior cingulate cortex BA24 (yellow); PCC23, posterior cingulate cortex BA23; SCC24/25, SCC BA24 and BA25 (red); vMF10, ventro-medial frontal cortex BA10.

(reviewed in ref. 13), provided an early anatomical perspective, consistently identifying clear abnormalities in both the frontal cortex and basal ganglia. Studies in individuals with primary depression, on the other hand, have indicated that structural abnormalities are more subtle, generally necessitating more advanced image acquisition and analytic approaches. Such studies have identified volume changes in the amygdala, hippocampus, and anterior cingulate, ventromedial, and prefrontal cortices but with considerable variability $(8,20-22)$ (Figure 1). Postmortem studies further identify glial cell loss in primary depression but the findings are also not localized to any one brain region (23-26). Unlike depression following a selective brain lesion or injury, in which causal inferences can be reasonably asserted, volume changes reported in MDD appear to be more complex, particularly since acute lesions of the amygdala, hippocampus, cingulate cortex, and ventromedial frontal cortex do not precipitate depressive symptoms or syndromes $(18,19)$. Furthermore, genetic risk factors and environmental stress may further contribute to some of these reported structural abnormalities, independent of the presence of depression (27-29). Nonetheless, comparative cytoarchitectural and anatomical connectivity studies generally confirm the critical involvement of these regions, most consistently the frontal cortex, cingulate cortex, basal ganglia, amygdala, and hippocampus in animal models of depression and associated emotional behaviors (30-34), supporting the hypothesis that even subtle disruption of pathways linking these regions in humans can result in disturbances in emotion regulation typical of MDD, namely negative mood coupled with sustained changes in motivation, motor performance, cognition, and circadian functions. Studies of regional brain dysfunction with functional imaging further support this hypothesis.
Functional imaging studies. There are now a variety of imaging methods (PET, single photon emission computed tomography, functional MRI [fMRI], magnetic resonance spectroscopy, EEG, magnetoencephalography, and optical imaging) capable of quantifying a wide range of physiological parameters relevant to the study of major depression. In this brief overview, resting-state blood flow and glucose metabolism measures using PET are highlighted, as they make up the bulk of the published studies. Functional imaging studies of primary depression (reviewed in refs. 6-8) commonly report frontal cortex and cingulate abnormalities, a pattern also seen in neurological depressions (13). Other limbic-paralimbic (amygdala, anterior temporal, and insula) and subcortical (basal ganglia and thalamus) abnormalities have also been identified, but the findings are more variable. Across studies, the most robust and best-replicated finding is that of decreased prefrontal cortex function, although normal frontal activity and frontal hyperactivity have also been reported $(35,36)$. Localization of abnormalities within the frontal lobe includes regions of the dorsolateral and ventral-lateral prefrontal cortex (specifically Brodmann area 9 [BA9], BA46, BA10, and BA47) as well as orbital frontal and ventromedial frontal cortices (specifically BA11, BA32, and BA10). Findings are generally bilateral, although asymmetries have been described. Cingulate cortex changes (increases or decreases in activity) are also commonly seen and consistently involve anterior sectors (in particular, BA24 and BA25).

\section{Potential sources of scan variability}

While there are clearly a highly reproducible set of functional and structural findings across studies, not all patients show the same pattern. Differences among patient subgroups (e.g., among those with familial, bipolar, unipolar, neurological, or early trauma depression) as well as heterogeneous expression of clinical symptoms, such as illness severity, cognitive impairment, anxiety, anhedonia, mood reactivity, and psychomotor slowing, are thought to contribute to the described variance, but there is not yet a consensus. The bestreplicated behavioral correlate of a resting-state abnormality in depression is that of an inverse relationship between the activity of the prefrontal cortex and the severity of depression. Low prefrontal cortex activity has also been correlated with slowed reaction times and impairment in cognitive functions such as attention and working memory; low parietal and parahippocampus activity has been associated with anxiety; medial frontal and cingulate hypoactivity has been associated with impairments in performance in error detection and set-shifting tasks; ventral striatum/nucleus accumbens hypoactivity has been associated with anhedonia; and amygdala hyperactivity has been associated with cortisol status (37-43). A more complex ventral-dorsal segregation of frontal lobe functions has also been described, with anxiety/tension positively correlated with ventral prefrontal cortex activity and psychomotor and cognitive slowing negatively correlated with dorsolateral prefrontal cortex activity (40). The prefrontal cortex and amygdala hyperactivity seen in patients with a more ruminative/anxious clinical presentation is also consistent with findings described in individuals with primary anxiety and obsessional disorders (39), memory-evoked anxiety and fear in healthy subjects and response to the testing environment due to novelty or state anxiety as well as gene-mediated variability in emotional reactivity (44). Even with these considerations, the presence of clinical symptom variability within a given patient cohort does not appear to fully explain the consistent inconsistencies in the published imaging literature $(6,17)$. 


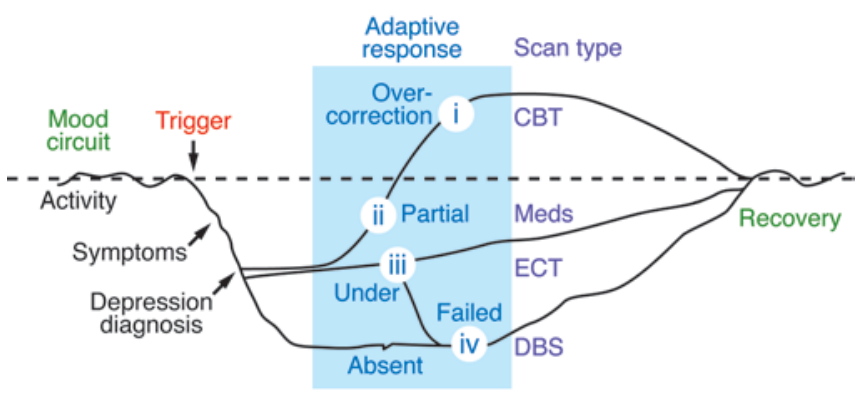

Figure 2

Theoretical time course of mood circuit changes during a depressive episode. Functional neuroimaging abnormalities are viewed as the net effect of a triggering event and subsequent intrinsic adaptive or maladaptive responses, in other words, failure to self-correct. The nature of these compensatory changes is considered critical for understanding clinical symptom heterogeneity and clinical subtypes of MDD, providing a potential future framework for the development of brain-based algorithms for treatment selection based on distinct circuit patterns or brain phenotypes (indicated here as scan types i-iv). By example, scan type i, characterized by maladaptive overcorrection of the circuit, might be optimally treated with CBT. In contrast, failure to initiate or sustain any adaptive response, as defined here by scan type iv, might require ECT or DBS.

One can alternatively consider variable patterns from a systems perspective, as outlined in Figure 2, in which dysregulated circuit activity identified in the baseline depressed state is seen to reflect both foci of primary dysfunction as well as sites of attempted (or failed) adaptation. Such a model would theoretically accommodate the reported variability among published depression cohorts, the recognized heterogeneity of depressive symptoms, and purported etiologic risk factors $(15,16)$ and is also in keeping with conceptual models of sustained allostatic load (45). Hypothetically, in the setting of sustained overactivity of the regulatory circuit (whatever the cause), an exaggerated or hypersensitive compensatory response may result in an agitated, mood-reactive, ruminative depressive state in one patient, whereas failure to initiate or maintain an adequate compensatory response may lead to anergy, psychomotor retardation, apathy, and mood nonreactivity in a second patient with equally severe depression. In this context, net circuit activity resulting in a sustained but only partially compensated state would probably respond equally well to either pharmacological or psychological treatments, consistent with empirical clinical experience as well as randomized controlled studies (46). On the other hand, more extreme states of adaptive changes in the circuit (either overactive or underactive) would require more specific treatments (i.e., CBT or interpersonal psychotherapy when overactive [ref. 47] and medication augmentation or ECT, vagus nerve stimulation, or repetitive transcranial magnetic stimulation when underactive [refs. 2, 48-51]), with failure of the circuit to initiate an adaptive response defining patients with TRD and a need for more aggressive interventions such as DBS $(11,12,52-54)$. Such hypotheses lay the foundation for a related goal, namely to define a specific brain circuit signature that could eventually provide a therapeutic road map for optimal treatment selection in individual depressed patients - if baseline variability and associated change patterns with different treatment interventions can be fully characterized (6). While important insights have been made using group-based analyses, as described in the following sections, practically speak- ing, fMRI may prove a more agile technology to achieve this goal, since circuit patterns, commonly termed "functional connectivity", are testable in individual subjects. Such strategies emphasize not merely the absolute state of regional activity, but rather, the way in which activities in different locales influence one another as indexed using region-region correlations or covariances (55-57).

\section{Scan variability as a biomarker of response likelihood}

In an attempt to define a neural signature that could indicate the treatment most likely to be effective in a given patient, several groups have already identified pretreatment scan patterns that differentiate response-specific subtypes to various treatments (58-67). Retrospective analyses of resting-state PET studies and, more recently, fMRI studies using behavioral tasks have consistently reported that increased pretreatment activity in the pregenual anterior cingulate cortex (pACC; BA24) distinguishes responders to several different antidepressant interventions from nonresponders $(58,59,63,67)$. However, prospective testing has not been done to indicate whether this pattern differentially predicts response to a specific treatment class. Using similar methods, SCC (specifically BA25) hyperactivity has been shown to predict response to sleep deprivation (64) and cingulotomy (66) in patients who had previously not responded to medication treatment, identifying a potential marker of treatment resistance $(11,62,68)$. Systematic characterization of these potential predictive patterns alone or in combination with genetic markers (69) has important therapeutic implications in light of increasing evidence that the presence of residual symptoms places patients at increased risk for future relapse or recurrence (48).

\section{Brain targets of antidepressant treatments}

As seen in studies of the baseline depressed state, PET measures of regional cerebral glucose metabolism and blood flow, and, more recently, resting-state and task fMRI have also proven to be sensitive indices of changing brain function following various treatments. Changes in cortical, limbic-paralimbic, and subcortical regions have been described following treatments as diverse as medication, psychotherapy, sleep deprivation, ECT, repetitive transcranial magnetic stimulation, vagus nerve stimulation, ablative surgery, and DBS $(6,7,10-12,62,70-78)$. While normalization of frontal abnormalities is the best-replicated finding, other regional effects are reported with variable patterns with different treatments. The nature of the specific imaging strategy and the behavior provocation or task used to test the effect of treatment contribute to variability. Despite this caveat, modality-specific effects are consistent with the hypothesis that different interventions modulate specific regional targets, resulting in a variety of complementary, adaptive chemical, and molecular changes sufficient to reestablish a euthymic, remitted state.

Medication. Across studies of chronic antidepressant treatment using commonly prescribed medications, prefrontal cortical changes are the most consistently reported, with normalization of frontal cortex overactivity and underactivity both described (62, 70-72). Additionally, changes are also seen in limbic and subcortical regions, including the subgenual cingulate, amygdala, hippocampus, posterior cingulate cortex, and insula, with decreased activity most commonly observed $(70,71,74-77)$. The time course of these medication effects and differences between responders and nonresponders have provided additional localizing clues as to critical brain changes mediating depression remission. In one such experiment (70), responders and nonresponders to the selective 
A SSRI

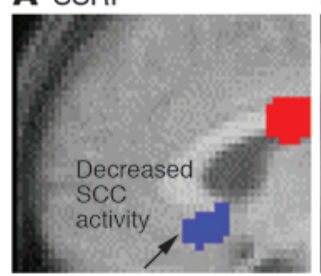

F $\mathrm{CBT} / \mathrm{NR}$

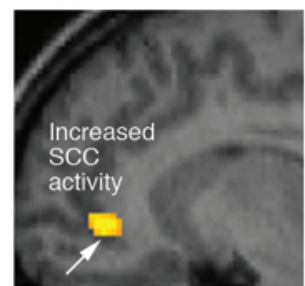

B Placebo

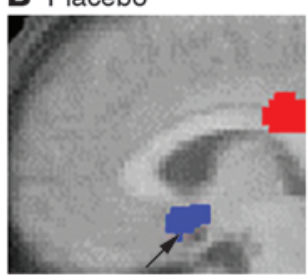

G Med NR

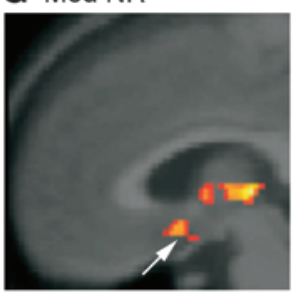

C SNRI

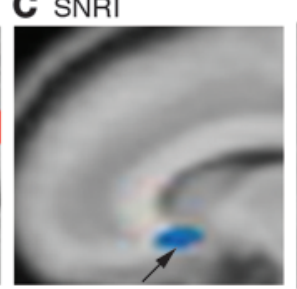

H TRD Pre-CGT

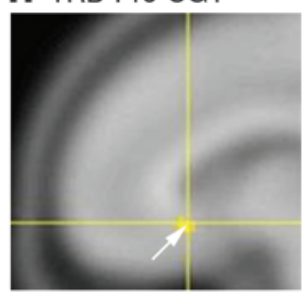

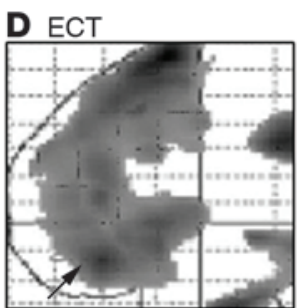

I TRD Pre-DBS

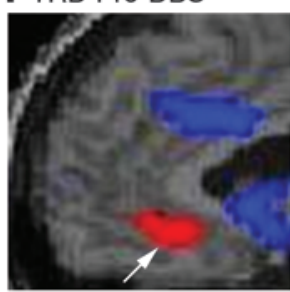

\section{E CBT}

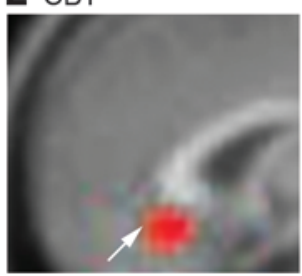

J Sad memory

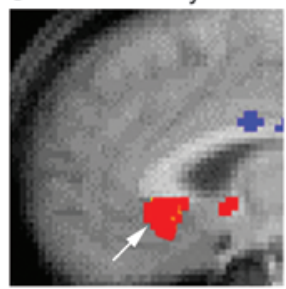

Figure 3

Converging evidence implicating the SCC region in MDD. (A-E) Common pattern of changes in glucose metabolism or blood flow in the SCC with antidepressant response to various interventions. Images demonstrate group change patterns relative to the baseline depressed state for each treatment: (A) metabolic decreases with the selective serotonin reuptake inhibitor (SSRI) fluoxetine; (B) metabolic decreases with a placebo pill; (C) metabolic decreases with the serotonin-norepinephrine reuptake inhibitor (SNRI) venlafaxine; (D) blood flow decreases with ECT; and (E) metabolic increases with CBT. (F-J) Images demonstrate elevated resting-state SCC25 activity in various groups of patients with TRD: (F) metabolic increases in CBT and venlafaxine (V) nonresponders (NRs) relative to both healthy subjects and similarly depressed patients who responded to either treatment; (G) resting-state fMRI increases in pharmacotherapy (Med) nonresponders relative to healthy controls; $(\mathbf{H})$ glucose metabolic increases in patients with TRD who later responded to cingulotomy (CGT) relative to those that failed to respond; (I) blood flow increases in patients with TRD, enrolled in a DBS treatment trial relative to healthy controls; (J) SCC blood flow increases with induction of transient sadness induced by recollection of a personal sad memory in healthy subjects, a pattern similar to that seen in patients with TRD. Red indicates increased activity (white arrows) and blue indicates decreased activity (black arrows). Images are courtesy of Mitch Nobler (D), Michael Greicius (G), and Darin Dougherty (H). Panels $\mathbf{A}$ and $\mathbf{J}$ are generated from data published in American Journal of Psychiatry (10). Panels $\mathbf{B}$ and $\mathbf{D}$ are adapted with permission from American Journal of Psychiatry (refs. 79 and 73, respectively). Panel I is adapted with permission from Neuron (11). Panels $\mathbf{C}$ and $\mathbf{E}$ are adapted with permission from American Journal of Psychiatry (62). Panel $\mathbf{F}$ is generated from data published in American Journal of Psychiatry (62). Panel $\mathbf{G}$ is adapted with permission from Biological Psychiatry (68). Panel $\mathbf{H}$ is adapted with permission from Journal of Neurosurgery (66).

serotonin reuptake inhibitor (SSRI) fluoxetine were differentiated by their pattern of glucose metabolism observed six weeks after the initiation of therapy, with clinical improvement associated with limbic-paralimbic and striatal decreases and dorsal cortical increase in glucose metabolism. Failed response was associated with persistence of the pattern of glucose metabolism observed one week after the initiation of therapy (when the same pattern was seen in both groups) and absence of either SCC (specifically BA25) or prefrontal cortex changes. This combination of reciprocal dorsal cortical and ventral limbic changes appears to be a common pattern in individuals that respond to treatment with SSRIs (72), placebo medication (79), and combination serotonin-norepinephrine reuptake inhibitors (SNRIs) (62). The change in the glucose metabolism pattern in only those patients who showed clinical improvement suggests a process of neural plasticity or adaptation in specific brain regions with chronic treatment. These responder-nonresponder differences are also consistent with the time course and location of changes identified in animal studies of antidepressant medications, which emphasize early brainstem and hippocampal changes and late cortical effects, involving presynaptic autoregulatory desensitization, up- and downregulation of multiple postsynaptic receptor sites, and receptor-mediated second messenger and neurotrophic intracellular signaling effects $(80,81)$.

Psychotherapy. In contrast to pharmacological treatments, CBT is thought to retrain brain activity by modifying attention and memory functions involved in the mediation of depression-relevant explicit cognitions, affective bias, and maladaptive information processing, all of which are putatively localized to orbital, medial frontal, prefrontal, and anterior cingulate regions of the cortex $(82,83)$. Imaging studies examining brain changes following interpersonal psychotherapy and CBT report substantial regional effects - most prominently decreased glucose metabolism and blood flow in the prefrontal cortex $(62,71,84,85)$, but with differential non-frontal cortex changes depending on the specific therapeutic intervention employed. For example, using CBT, remission was associated with not only prefrontal cortex changes but also decreased metabolism in posterior cingulate, dorsomedial frontal, and orbital frontal cortices as well as increased metabolism in anterior mid-cingulate cortex and parahippocampal regions (71). This CBT-specific change pattern was generally replicated in a follow-up, randomized study comparing treatment with CBT and the SNRI venlafaxine (62). Interestingly, this second study identified both a common decrease in dorsomedial frontal cortical activity with both treatments as well as reciprocal changes in subgenual cingulate cortex activity (increased with CBT, decreased, as seen previously, with medication), further suggesting a critical role for this region in mediating remission from depression across treatments.

\section{Critical role for the SCC}

Among the series of treatment studies surveyed, the involvement of the SCC is especially prominent (Figure 3, A-E). Not only do changes in this region appear critical for antidepressant response 


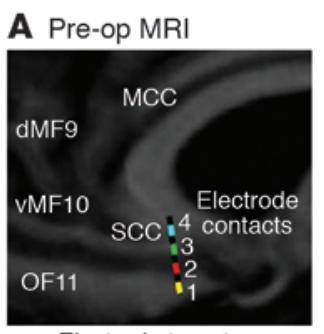

Electrode target: SCC white matter

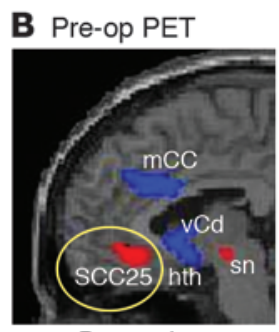

Depression:

Overactive SCC25

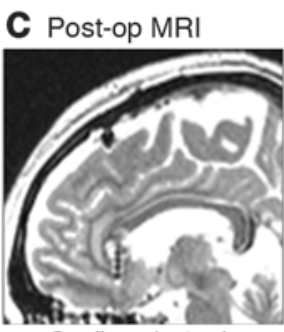

Confirm electrode placement

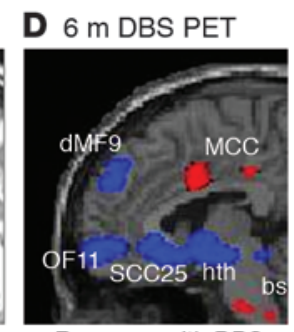

Recovery with DBS SCC25 suppression

Figure 4

Selective targeting of the depression circuit with DBS in patients with TRD. (A) Preoperative (pre-op) MRI demonstrating the intended anatomical location for the DBS electrode within the SCC white matter. The four individual contacts on the electrode (numbered 1-4) can be independently stimulated using a programmable implanted pulse generator. (B) Preoperative blood flow PET scan demonstrating baseline hyperactivity of the SCC in the TRD study group $(n=6)$ relative to healthy controls. (C) The postoperative (post-op) MRI with the electrodes in place within the SCC white matter. (D) Six-month blood flow change relative to preoperative baseline associated with chronic DBS of the optimal SCC contact in four DBS responders. Red indicates increased blood flow and blue indicates decreased blood flow. hth, hypothalamus; MCC, mid-cingulate cortex; sn, substantia nigra; vCd, ventral caudate (adapted with permission from Neuron [ref. 11]).

to active and placebo pharmacotherapy, ECT, and CBT $(62,70$, $73,79)$, but functional hyperactivity of this region best characterizes more treatment-resistant patients (Figure 3, F-I) $(11,62$, $65,66,68)$. Furthermore, anatomical changes on structural MRI scans as well as postmortem identification of glial cell abnormalities $(21,23)$ are reported in depressed patient samples. In addition, structural and functional variability in this region has been linked to a normal polymorphism in the serotonin transporter, an emerging risk factor for depression (28). These converging anatomical findings complement a large functional imaging literature, linking the SCC to the regulation of negative emotional states, as illustrated by increased activity in this region with the provocation of sad mood, using either autobiographical memory (Figure 3J) (10) or a pharmacological challenge, such as tryptophan depletion (86), as well as passive exposure to sad, negative, or unpleasant pictures and words $(61,87)$.

The foundation for a more specific role of the SCC in the autonomic and circadian aspects of emotion regulation, as occurs with both stress and depression, including alterations in sleep, appetite, libido, and endocrine functioning, is also suggested by the predominant afferent and efferent connections of the SCC to the insula, brainstem, and hypothalamus $(34,88-91)$. Reciprocal pathways linking SCC BA25 to orbitofrontal, medial frontal, and dorsal prefrontal cortices, the anterior and posterior cingulate cortices, and to the amygdala, hippocampus, and nucleus accumbens further identify plausible pathways by which interceptive and homeostatic processes might influence aspects of learning, memory, reward, and reinforcement $(34,91-94)$, which are core behaviors impaired in depressed patients. Interestingly, these various connections show considerable overlap with the pattern of regional changes seen with both CBT and pharmacotherapy treatment described above, providing strong evidence to pursue strategies that might effectively alter SCC connectivity in individuals with TRD, rather than focusing on merely correcting absolute activity in SCC in isolation.

\section{Testing the model: targeting the SCC with DBS}

The repeated observations of increased activity in the SCC in studies of acute negative affective states (sadness), cellular abnormalities in this region in depressed patients postmortem, and predictable decreases in activity with a variety of pharmacological and somatic antidepressant treatments provided the critical foundation to test the use of direct modulation of SCC (BA25), using high frequency DBS as a novel treatment strategy for individuals with otherwise treatment-resistant MDD. Based on the well-established evidence in Parkinson disease (PD), demonstrating that chronic, high frequency DBS in pathologically overactive motor circuits produces profound clinical benefits (95), it was hypothesized that focal stimulation of the SCC and adjacent white matter would not only reduce chronically elevated SCC activity but would also normalize aberrant activity throughout the depression circuit, with resulting clinical benefit.

In a proof-of-principle study, six patients with refractory MDD who had failed to respond to multiple medications as well as psychotherapy and ECT were implanted with two DBS electrodes (one in each hemisphere) under local anesthesia, using MRI guidance and stereotaxic surgical procedures routinely used in the treatment of PD. A protocol similar to that used in evaluating stimulation thresholds for efficacy and adverse effects for PD was also adopted for use in the patients with TRD, with stimulation settings programmed via an implanted pulse generator inserted in the subcutaneous chest wall. Chronic bilateral stimulation (pulse width $=90 \mu \mathrm{s}$; frequency $=130 \mathrm{~Hz}$; voltage $\cong 4 \mathrm{~V}$ ) of the white matter tracts adjacent to the SCC produced a sustained remission of depressive symptoms in four of the six patients in the pilot study (11). Consistent with the circuit hypothesis stated above, clinical antidepressant effects were associated with a marked reduction in SCC blood flow as well as changes in downstream limbic and cortical sites (decreased activity in the hypothalamus, ventral striatum, and orbital and medial frontal cortices and increased activity in the dorsolateral prefrontal, parietal, mid-cingulate, and posterior cingulate cortices) measured using PET (Figure 4). While it is not yet clear which of these remote changes is most critical to the sustained antidepressant effects, the clinical and imaging findings have now been replicated in an additional 14 patients, with an overall six-month response rate of $60 \%$ and with sustained response exceeding one year (12). Placebo-controlled trials extending these first studies are now underway, examining both clinical efficacy and mechanisms as well as potential clinical or imaging biomarkers that might identify the patients with TRD most likely to benefit from this intervention. 


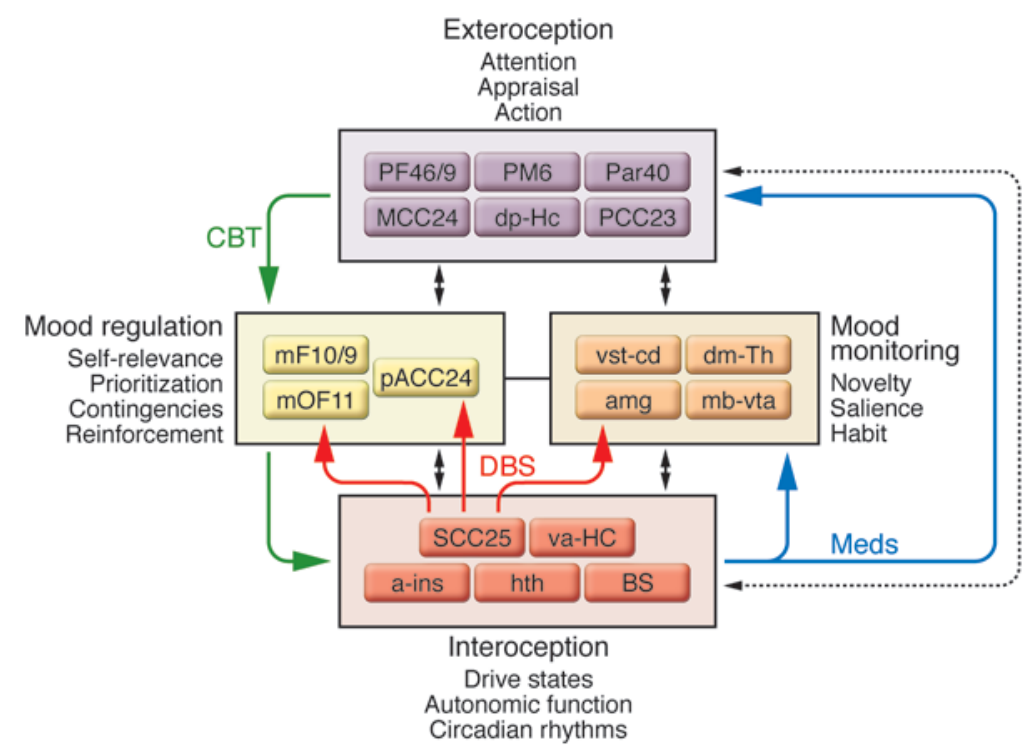

Figure 5

Circuit model of MDD. Regions with known anatomical interconnections that show consistent changes across converging imaging experiments form the basis of this model. Regions are grouped into four main compartments, reflecting general behavioral dimensions of MDD and regional targets of various antidepressant treatments. Regions within a compartment all have strong anatomical connections to one another. Black arrows identify cross-compartment anatomical connections. Solid colored arrows identify putative connections between compartments mediating a specific treatment: green indicates CBT; blue indicates pharmacotherapy; red indicates SCC DBS. a-ins, anterior insula; amg, amygdala; dm-Th, dorsomedial thalamus; dp-Hc, dorsal-posterior hippocampus; mb-vta, midbrain-ventral tegmental area; $\mathrm{mF} 10 / 9$, medial frontal cortex BA10 and BA9; mOF11, medial orbital frontal cortex BA11; Par40, parietal cortex BA40; PF46/9, prefrontal cortex BA46 and BA9; PM6, premotor cortex BA6; va-HC, ventral-anterior hippocampus; vst-cd, ventral striatum-caudate.

\section{Emergent questions}

In addition to the apparent sustained rate of clinical recovery in the first experimental group treated with DBS was the intriguing observation that the precise target of stimulation was extremely critical, with variable clinical effects seen with stimulation of adjacent contacts separated by mere millimeters along the same electrode (Figure 4, left panel). Similarly, with patients awake in the operating room, immediate behavioral effects were often seen with acute stimulation of some but not all the individual contacts. Such acute effects often predicted future sustained antidepressant response with chronic stimulation at the same contact. Among spontaneous patient reports, "a lifting of the void" or resolution of dread were most common, with heightened interest and a sense of "connectedness" as well as increased motor speed, increased volume and rate of spontaneous speech, and improved prosody also observed (11). These provocative findings have not been systematically characterized either clinically or with appropriate imaging strategies but are the focus of current experiments. New studies have also implemented diffusion imaging and probabilistic tractography analyses and are now examining the specific white matter tracts affected by focal stimulation of adjacent individual contacts to further delineate which pathways mediate these potentially critical acute stimulation effects $(96,97)$. Such studies combined with real-time electrophysiological recordings in animals will provide new models that inform on system-level dynamics, mediating the transition from acute to sustained antidepressant effects at the cellular, local circuit, and network level.

\section{Unifying depression circuit model}

To help summarize converging findings and facilitate future experiments, an expanded version of a previously proposed and evolving multi-node circuit model of depression $(6,9)$ has been constructed from the various studies discussed in the previous sections (Figure 5). Regions within this model are clustered into four main functional compartments, based on reproducible patterns across various experiments, and informed by comparative anatomical, electrophysiological, and tract-tracing experiments in nonhuman primates and rodents (34, 88-94, 98). Such compartmental groupings aim to accommodate the major defining symptoms of MDD (sustained mood, motor, cognitive, and circadian dysfunction) and changes in these behaviors accompanying treatment and recovery. The model further attempts to capture basic cognitive (exteroceptive) and visceral-motor (interoceptive) processes mediating normal responses to novel overt and covert emotional stimuli (34, 38, 44, 99-108), recognizing that the complexities and nuances of brain mechanisms mediating these behaviors are grossly oversimplified by this model. With this caveat, consistent functional interactions and region-region correlations are emphasized in the assignment of a particular region to a specific compartment, with explicit anatomical connections linking individual regions both within and across compartments critical to the core model construct.

While earlier versions of this model implicated a more limited set of brain regions $(9,13)$, what has remained consistent across models are the reciprocal interactions between ventral limbic (interoceptive) regions and dorsal cortical (exteroceptive) regions as a hallmark of a negative mood state (increased limbic activity and decreased cortical activity), occurring either transiently - such as with an acute emotional provocation - or when sustained, as seen during a major depressive episode. Similarly, reversal of this pattern (decreased limbic activity and increased cortical activity) characterizes mood improvement, with depression remission (10, 11). Medial frontal cortical regions have been assigned a distinct compartment in the model, as these regions appear most critical to active cognitive control and overt regulation of emotional and affective state $(37,103-106,109)$. Similarly, a set of subcortical regions, including the amygdala, basal ganglia, and thalamus, which have been consistently implicated in primary and often covert processing of novel emotional and nonemotional stimuli (37), have been grouped together to emphasize their more general role in evaluating salience (107) and in mediating reinforcement, learning, and habit (38, 44, 108, 110-112). Ventral (subcallosal SCC BA25), rostral (pACC BA24), and dorsal (supragenual or midcingulate cortex BA24) subregions of the anterior cingulate cortex (also shown in Figure 1) have been similarly segregated, in keeping with their differential anatomical connections within and between 
compartments $(34,88,94,98)$. Within this framework, synchronized changes within and across compartments are considered critical for illness remission, regardless of treatment modality, accommodating the described pharmacotherapy as well as cognitive and surgical interventions. That said, it is not yet clear if the specific balance between regions or compartments is differentially sensitive to a particular treatment, as postulated in Figure 2, but this remains an important focus of ongoing studies.

\section{Future directions}

Multicenter, randomized, placebo-controlled trials are ultimately necessary to determine the clinical efficacy of DBS for TRD, using modulation of the SCC as described here $(11,12)$ or modulation of other brain regions currently being testing, including the anterior limb of the internal capsule, the nucleus accumbens, and the inferior thalamic peduncle (52-54). That said, complementary research studies in patients undergoing these procedures offer unique opportunities to examine both disease and treatment mechanisms from new perspectives. Such studies will optimally benefit from a flexible research infrastructure that can take best advantage of ongoing advances in multimodal imaging and computational modeling of complex circuits $(109,113-116)$ as well as new animal models (30-32, 117-119). As has proven to be the case with DBS for the treatment of PD (120-124), such platforms facili- tate the necessary translational studies needed to fully characterize DBS effects at the cellular, molecular, and network levels. It is further expected that continued refinement of brain-based circuit models for depression and other neuropsychiatric disorders will have a growing role not only in the development of novel therapies but in defining a new depression nosology, a critical step toward the eventual use of evidence-based brain biomarkers to optimize treatment selection in individual patients.

\section{Acknowledgments}

I thank my many colleagues who contributed to these many studies, including Robert Robinson, Sergio Starkstein, Stephen Brannan, Mario Liotti, Sidney Kennedy, Zindel Segal, Andres Lozano, Paul Holtzheimer, and Cameron Craddock. Past and current work is supported by grants from the National Institute of Mental Health (NIMH), Canadian Institute for Health Research (CIHR), National Alliance for Research on Schizophrenia and Depression (NARSAD), Dana Foundation, Stanley Medical Research Institute, and the Woodruff Foundation.

Address correspondence to: Helen Mayberg, Emory University, Department of Psychiatry, 101 Woodruff Circle, WMB 4313, Atlanta, Georgia 30322, USA. Phone: (404) 727-6740; Fax: (404) 727-6743; E-mail: hmayber@emory.edu.
1. McKenna, M.T., Michaud, C.M., Murray, C.J., and Marks, J.S. 2005. Assessing the burden of disease in the United States using disability-adjusted life years. Am. J. Prev. Med. 28:415-423.

2. Warden, D., Rush, A.J., Trivedi, M.H., Fava, M., and Wisniewski, S.R. 2007. The STAR*D Project results: a comprehensive review of findings. Curr. Psychiatry Rep. 9:449-459.

3. Holtzheimer, P.E., and Nemeroff, C.B. 2008. Novel targets for antidepressant therapies. Curr. Psychiatry Rep. 10:465-473.

4. Davidson, R.J., Pizzagalli, D., Nitschke, J.B., and Putnam, K. 2002. Depression: perspectives from affective neuroscience. Annu. Rev. Psychol. 53:545-574.

5. Phillips, M.L., Drevets, W.C., Rauch, S.L., and Lane, R. 2003. Neurobiology of emotion perception II: Implications for major psychiatric disorders. Biol. Psychiatry. 54:515-528.

6. Mayberg, H.S. 2003. Modulating dysfunctional limbic-cortical circuits in depression: towards development of brain-based algorithms for diagnosis and optimised treatment. Br. Med. Bull. 65:193-207.

7. Fitzgerald, P.B., Laird, A.R., Maller, J., and Daskalakis, Z.J. 2008. A meta-analytic study of changes in brain activation in depression. Hum. Brain Mapp. 29:683-695.

8. Drevets, W.C., Price, J.L., and Furey, M.L. 2008. Brain structural and functional abnormalities in mood disorders: implications for neurocircuitry models of depression. Brain Struct. Funct. 213:93-118.

9. Mayberg, H.S. 1997. Limbic-cortical dysregulation: a proposed model of depression. J. Neuropsychiatry Clin. Neurosci. 9:471-481.

10. Mayberg, H.S., et al. 1999. Reciprocal limbic-cortical function and negative mood: converging PET findings in depression and normal sadness. Am.J. Psychiatry. 156:675-682.

11. Mayberg, H.S., et al. 2005. Deep brain stimulation for treatment-resistant depression. Neuron. 45:651-660.

12. Lozano, A.M., et al. 2008. Subcallosal cingulate gyrus deep brain stimulation for treatment-resistant depression. Biol. Psychiatry. 64:461-467.

13. Mayberg, H.S. 1994. Frontal lobe dysfunction in secondary depression. J. Neuropsychiatry Clin. Newrosci. 6:428-442.
14. McEwen, B.S., and Seeman, T. 1999. Protective and damaging effects of mediators of stress. Elaborating and testing the concepts of allostasis and allostatic load. Ann. N. Y. Acad. Sci. 896:30-47.

15. Kendler, K.S., Thornton, L.M., and Gardner, C.O. 2001. Genetic risk, number of previous depressive episodes, and stressful life events in predicting onset of major depression. Am. J. Psychiatry. 158:582-586

16. Caspi, A., et al. 2003. Influence of life stress on depression: moderation by a polymorphism in the 5-HTT gene. Science. 301:386-389.

17. Hasler, G., Drevets, W.C., Manji, H.K., and Charney, D.S. 2004. Discovering endophenotypes for major depression. Neuropsychopharmacology. 29:1765-1781.

18. Robinson, R.G., Kubos, K.L., Starr, L.B., Rao, K., and Price, T.R. 1984. Mood disorders in stroke patients. Importance of location of lesion. Brain. 107:81-93.

19. Koenigs, M., et al. 2008. Distinct regions of prefrontal cortex mediate resistance and vulnerability to depression. J. Neurosci. 28:12341-12348.

20. Sheline, Y.I. 2003. Neuroimaging studies of mood disorder effects on the brain. Biol. Psychiatry. 54:338-352.

21. Drevets, W.C., et al. 1997. Subgenual prefrontal cortex abnormalities in mood disorders. Nature. 386:824-827.

22. Pizzagalli, D.A., et al. 2004. Functional but not structural subgenual prefrontal cortex abnormalities in melancholia. Mol. Psychiatry. 9:325, 395-405.

23. Ongur, D., Drevets, W.C., and Price, J.L. 1998. Glial reduction in the subgenual prefrontal cortex in mood disorders. Proc. Natl. Acad. Sci. U. S. A. 95:13290-13295.

24. Bowley, M.P., Drevets, W.C., Ongur, D., and Price, J.L. 2002. Low glial numbers in the amygdala in major depressive disorder. Biol. Psychiatry. 52:404-412.

25. Harrison, P.J. 2002. The neuropathology of primary mood disorder. Brain. 125:1428-1449.

26. Rajkowska, G., and Miguel-Hidalgo, J.J. 2007. Gliogenesis and glial pathology in depression. CNSNeurol. Disord. Drug Targets. 6:219-233.

27. McEwen, B.S., and Magarinos, A.M. 1997. Stress effects on morphology and function of the hippocampus. Ann. N. Y. Acad. Sci. 821:271-284.
28. Pezawas, L., et al. 2005. 5-HTTLPR polymorphism impacts human cingulate-amygdala interactions: a genetic susceptibility mechanism for depression. Nat. Neurosci. 8:828-834.

29. Radley, J.J., et al. 2008. Repeated stress alters dendritic spine morphology in the rat medial prefrontal cortex. J. Comp. Neurol. 507:1141-1150.

30. Banasr, M., and Duman, R.S. 2007. Regulation of neurogenesis and gliogenesis by stress and antidepressant treatment. CNS Neurol. Disord. Drug Targets. 6:311-320.

31. Krishnan, V., and Nestler, E.J. 2008. The molecular neurobiology of depression. Nature. 455:894-902.

32. Airan, R.D., et al. 2007. High-speed imaging reveals neurophysiological links to behavior in an animal model of depression. Science. 317:819-823.

33. Diorio, D., Viau, V., and Meaney, M.J. 1993. The role of the medial prefrontal cortex (cingulate gyrus) in the regulation of hypothalamic-pituitary-adrenal responses to stress. J. Neurosci. 13:3839-3847.

34. Barbas, H., Saha, S., Rempel-Clower, N., and Ghashghaei, T. 2003. Serial pathways from primate prefrontal cortex to autonomic areas may influence emotional expression. BMC Neurosci. 4:25.

35. Baxter, L.R., Jr., et al. 1985. Cerebral metabolic rates for glucose in mood disorders. Studies with positron emission tomography and fluorodeoxyglucose F 18. Arch. Gen. Psychiatry. 42:441-447.

36. Drevets, W.C., et al. 1992. A functional anatomical study of unipolar depression. J. Neurosci. 12:3628-3641.

37. Wang, L., et al. 2008. Prefrontal mechanisms for executive control over emotional distraction are altered in major depression. Psychiatry Res. 163:143-155.

38. Tremblay, L.K., et al. 2005. Functional neuroanatomical substrates of altered reward processing in major depressive disorder revealed by a dopaminergic probe. Arch. Gen. Psychiatry. 62:1228-1236.

39. Osuch, E.A., et al. 2000. Regional cerebral metabolism associated with anxiety symptoms in affective disorder patients. Biol. Psychiatry. 48:1020-1023.

40. Brody, A.L., et al. 2001. Brain metabolic changes associated with symptom factor improvement in major depressive disorder. Biol. Psychiatry. 50:171-178.

41. Dunn, R.T., et al. 2002. Principal components of 
the Beck Depression Inventory and regional cerebral metabolism in unipolar and bipolar depression. Biol. Psychiatry. 51:387-399.

42. Drevets, W.C., et al. 2002. Glucose metabolism in the amygdala in depression: relationship to diag nostic subtype and plasma cortisol levels. Pharmacol. Biochem. Behav. 71:431-447.

43. Videbech, P., et al. 2002. The Danish PET/depression project: clinical symptoms and cerebral blood flow. A regions-of-interest analysis. Acta. Psychiatr. Scand. 106:35-44.

44. Hariri, A.R., et al. 2005. A susceptibility gene for affective disorders and the response of the human amygdala. Arch. Gen. Psychiatry. 62:146-152.

45. McEwen, B.S. 1998. Protective and damaging effects of stress mediators. N. Engl. J. Med. 338:171-179.

46. DeRubeis, R.J., et al. 2005. Cognitive therapy vs medications in the treatment of moderate to severe depression. Arch. Gen. Psychiatry. 62:409-416.

47. Nemeroff, C.B., et al. 2003. Differential responses to psychotherapy versus pharmacotherapy in patients with chronic forms of major depression and childhood trauma. Proc. Natl. Acad. Sci. U. S. A 100:14293-14296.

48. Rush, A.J., et al. 2006. Acute and longer-term outcomes in depressed outpatients requiring one or several treatment steps: a STAR*D report. Am. J. Psychiatry. 163:1905-1917.

49. Kellner, C.H., et al. 2006. Continuation electroconvulsive therapy vs pharmacotherapy for relapse prevention in major depression: a multisite study from the Consortium for Research in Electroconvulsive Therapy (CORE). Arch. Gen. Psychiatry. 63:1337-1344

50. Lam, R.W., Chan, P., Wilkins-Ho, M., and Yatham, L.N. 2008. Repetitive transcranial magnetic stimulation for treatment-resistant depression: a systematic review and metaanalysis. Can. J. Psychiatry. 53:621-631.

51. Nierenberg, A.A., Alpert, J.E., Gardner-Schuster, E.E., Seay, S., and Mischoulon, D. 2008. Vagus nerve stimulation: 2-year outcomes for bipolar versus unipolar treatment-resistant depression. Biol. Psychiatry. 64:455-460.

52. Schlaepfer, T.E., et al. 2008. Deep brain stimulation to reward circuitry alleviates anhedonia in refractory major depression. Neuropsychopharmacology. 33:368-377.

53. Malone, D.A., Jr., et al. 2008. Deep brain stimulation of the ventral capsule/ventral striatum for treatment-resistant depression. Biol. Psychiatry. 65:267-275

54. Jimenez, F., et al. 2005. A patient with a resistant major depression disorder treated with deep brain stimulation in the inferior thalamic peduncle. Neurosurgery. 57:585-593; discussion 585-593.

55. Friston, K., Phillips, J., Chawla, D., and Buchel, C. 1999. Revealing interactions among brain systems with nonlinear PCA. Hum. Brain Mapp. 8:92-97.

56. Horwitz, B. 2004. Relating fMRI and PET signals to neural activity by means of large-scale neural models. Neuroinformatics. 2:251-266.

57. Margulies, D.S., et al. 2007. Mapping the functional connectivity of anterior cingulate cortex. Neuroimage. 37:579-588.

58. Mayberg, H.S., et al. 1997. Cingulate function in depression: a potential predictor of treatment response. Neuroreport. 8:1057-1061.

59. Pizzagalli, D., et al. 2001. Anterior cingulate activity as a predictor of degree of treatment response in major depression: evidence from brain electrical tomography analysis. Am. J. Psychiatry. 158:405-415.

60. Little, J.T., et al. 1996. Venlafaxine or bupropion responders but not nonresponders show baseline prefrontal and paralimbic hypometabolism compared with controls. Psychopharmacol. Bull.
32:629-635.

61. Siegle, G.J., Carter, C.S., and Thase, M.E. 2006. Use of FMRI to predict recovery from unipolar depression with cognitive behavior therapy. Am. J. Psychiatry. 163:735-738

62. Kennedy, S.H., et al. 2007. Differences in brain glucose metabolism between responders to CBT and venlafaxine in a 16 -week randomized controlled trial. Am. J. Psychiatry. 164:778-788.

63. Chen, C.H., et al. 2007. Brain imaging correlates of depressive symptom severity and predictors of symptom improvement after antidepressant treatment. Biol. Psychiatry. 62:407-414.

64. Wu, J., et al. 1999. Prediction of antidepressant effects of sleep deprivation by metabolic rates in the ventral anterior cingulate and medial prefrontal cortex. Am. J. Psychiatry. 156:1149-1158.

65. Seminowicz, D.A., et al. 2004. Limbic-frontal circuitry in major depression: a path modeling metanalysis. Neuroimage. 22:409-418.

66. Dougherty, D.D., et al. 2003. Cerebral metabolic correlates as potential predictors of response to anterior cingulotomy for treatment of major depression. J. Neurosurg. 99:1010-1017.

67. Salvadore, G., et al. 2009. Increased anterior cingulate cortical activity in response to fearful faces: a neurophysiological biomarker that predicts rapid antidepressant response to ketamine. Biol. Psychiatry. 65:289-295.

68. Greicius, M.D., et al. 2007. Resting-state functional connectivity in major depression: abnormally increased contributions from subgenual cingulate cortex and thalamus. Biol. Psychiatry. 62:429-437.

69. Eichelbaum, M., Ingelman-Sundberg, M., and Evans, W.E. 2006. Pharmacogenomics and individualized drug therapy. Annu. Rev. Med. 57:119-137.

70. Mayberg, H.S., et al. 2000. Regional metabolic effects of fluoxetine in major depression: serial changes and relationship to clinical response. Biol. Psychiatry. 48:830-843.

71. Goldapple, K., et al. 2004. Modulation of corticallimbic pathways in major depression: treatmentspecific effects of cognitive behavior therapy. Arch. Gen. Psychiatry. 61:34-41.

72. Kennedy, S.H., et al. 2001. Changes in regional brain glucose metabolism measured with positron emission tomography after paroxetine treatment of major depression. Am. J. Psychiatry. 158:899-905.

73. Nobler, M.S., et al. 2001. Decreased regional brain metabolism after ECT. Am. J. Psychiatry. 158:305-308.

74. Pardo, J.V., et al. 2008. Chronic vagus nerve stimulation for treatment-resistant depression decreases resting ventromedial prefrontal glucose metabolism. Neuroimage. 42:879-889.

75. Sheline, Y.I., et al. 2001. Increased amygdala response to masked emotional faces in depressed subjects resolves with antidepressant treatment: an fMRI study. Biol. Psychiatry. 50:651-658.

76. Fu, C.H., et al. 2004. Attenuation of the neural response to sad faces in major depression by antidepressant treatment: a prospective, event-related functional magnetic resonance imaging study. Arch. Gen. Psychiatry. 61:877-889.

77. Anand, A., et al. 2005. Antidepressant effect on connectivity of the mood-regulating circuit: an FMRI study. Neuropsychopharmacology. 30:1334-1344

78. Luborzewski, A., et al. 2007. Metabolic alternations in the dorsolateral prefrontal cortex after treatment with high-frequency repetitive transcrania magnetic stimulation in patients with unipolar depression. J. Psychiatr. Res. 41:606-615.

79. Mayberg, H.S., et al. 2002. The functional neuroanatomy of the placebo effect. Am. J. Psychiatry. 159:728-737.

80. Freo, U., Ori, C., Dam, M., Merico, A., and Pizzolato, G. 2000. Effects of acute and chronic treatment with fluoxetine on regional glucose cerebral metab- olism in rats: implications for clinical therapies. Brain Res. 854:35-41.

81. Frechilla, D., Otano, A., and Del Rio, J. 1998. Effect of chronic antidepressant treatment on transcription factor binding activity in rat hippocampus and frontal cortex. Prog. Neuropsychopharmacol. Biol. Psychiatry. 22:787-802.

82. Watkins, E., and Teasdale, J.D. 2004. Adaptive and maladaptive self-focus in depression. J. Affect. Disord. $82: 1-8$

83. Rush, A.J., Kovacs, M., Beck, A.T., Weissenburger, J., and Hollon, S.D. 1981. Differential effects of cognitive therapy and pharmacotherapy on depressive symptoms. J. Affect. Disord. 3:221-229.

84. Brody, A.L., et al. 2001. Regional brain metabolic changes in patients with major depression treated with either paroxetine or interpersonal therapy: preliminary findings. Arch. Gen. Psychiatry. 58:631-640.

85. Martin, S.D., Martin, E., Rai, S.S., Richardson, M.A., and Royall, R. 2001. Brain blood flow changes in depressed patients treated with interpersonal psychotherapy or venlafaxine hydrochloride: preliminary findings. Arch. Gen. Psychiatry. 58:641-648.

86. Talbot, P.S., and Cooper, S.J. 2006. Anterior cingulate and subgenual prefrontal blood flow changes following tryptophan depletion in healthy males. Neuropsychopharmacology. 31:1757-1767.

87. Zald, D.H., Mattson, D.L., and Pardo, J.V. 2002. Brain activity in ventromedial prefrontal cortex correlates with individual differences in negative affect. Proc. Natl. Acad. Sci. U. S. A. 99:2450-2454.

88. Ghashghaei, H.T., Hilgetag, C.C., and Barbas, H. 2007. Sequence of information processing for emotions based on the anatomic dialogue between prefrontal cortex and amygdala. Neuroimage. 34:905-923.

89. Hsu, D.T., and Price, J.L. 2007. Midline and intralaminar thalamic connections with the orbital and medial prefrontal networks in macaque monkeys. J. Comp. Neurol. 504:89-111.

90. Freedman, L.J., Insel, T.R., and Smith, Y. 2000. Subcortical projections of area 25 (subgenual cortex) of the macaque monkey. J. Comp. Neurol. 421:172-188.

91. Vertes, R.P. 2004. Differential projections of the infralimbic and prelimbic cortex in the rat. Synapse. $51: 32-58$

92. Haber, S.N., Kim, K.S., Mailly, P., and Calzavara, R 2006. Reward-related cortical inputs define a large striatal region in primates that interface with associative cortical connections, providing a substrate for incentive-based learning. J. Neurosci. 26:8368-8376.

93. Ongur, D., and Price, J.L. 2000. The organization of networks within the orbital and medial prefrontal cortex of rats, monkeys and humans. Cereb. Cortex. 10:206-219.

94. Petrides, M., and Pandya, D.N. 2007. Efferent association pathways from the rostral prefrontal cortex in the macaque monkey. J. Neurosci. 27:11573-11586.

95. Benabid, A.L. 2003. Deep brain stimulation for Parkinson's disease. Curr. Opin. Neurobiol. 13:696-706.

96. Johansen-Berg, H., et al. 2008. Anatomical connectivity of the subgenual cingulate region targeted with DBS for treatment-resistant depression. Cereb. Cortex. 18:1374-1383.

97. Gutman, D.A., Holtzheimer, P.E., Behrens, T.E., Johansen-Berg, H., and Mayberg, H.S. 2008. A tractography analysis of two deep brain stimulation white matter targets for depression. Biol. Psychiatry. 65:276-282.

98. Vogt, B.A., Nimchinsky, E.A., Vogt, L.J., and Hof, P.R. 1995. Human cingulate cortex: surface features, flat maps, and cytoarchitecture. J. Comp. Neurol. 359:490-506

99. Craig, A.D. 2002. How do you feel? Interoception: the sense of the physiological condition of the 
body. Nat. Rev. Neurosci. 3:655-666.

100. Northoff, G., et al. 2006. Self-referential processing in our brain--a meta-analysis of imaging studies on the self. Neuroimage. 31:440-457.

101.Phan, K.L., Wager, T., Taylor, S.F., and Liberzon, I. 2002. Functional neuroanatomy of emotion: a meta-analysis of emotion activation studies in PET and PMRI. Neuroimage. 16:331-348.

102. Harvey, P.O., et al. 2005. Cognitive control and brain resources in major depression: an fMRI study using the n-back task. Neuroimage. 26:860-869.

103.Etkin, A., Egner, T., Peraza, D.M., Kandel, E.R., and Hirsch, J. 2006. Resolving emotional conflict: a role for the rostral anterior cingulate cortex in modulating activity in the amygdala. Neuron. 51:871-882.

104.Fossati, P., et al. 2003. In search of the emotional self: an FMRI study using positive and negative emotional words. Am. J. Psychiatry. 160:1938-1945.

105. Yoshimura, S., et al. 2009. Self-referential processing of negative stimuli within the ventral anterior cingulate gyrus and right amygdala. Brain Cogn. 69:218-225.

106. Ochsner, K.N., et al. 2004. For better or for worse: neural systems supporting the cognitive down- and up-regulation of negative emotion. Neuroimage. 23:483-499.

107.Zink, C.F., Pagnoni, G., Martin, M.E., Dhamala, M., and Berns, G.S. 2003. Human striatal response to salient nonrewarding stimuli. J. Neurosci. 23:8092-8097.

108.Siegle, G.J., Steinhauer, S.R., Thase, M.E., Stenger, V.A., and Carter, C.S. 2002. Can't shake that feeling: event-related fMRI assessment of sustained amygdala activity in response to emotional information in depressed individuals. Biol. Psychiatry. 51:693-707.

109. Koechlin, E., Ody, C., and Kouneiher, F. 2003. The architecture of cognitive control in the human prefrontal cortex. Science. 302:1181-1185.

110.Knutson, B., and Greer, S.M. 2008. Anticipatory affect: neural correlates and consequences for choice. Philos. Trans. R Soc. Lond. B Biol. Sci. 363:3771-3786.

111.Myers, K.M., and Davis, M. 2007. Mechanisms of fear extinction. Mol. Psychiatry. 12:120-150.

112.Santini, E., Quirk, G.J., and Porter, J.T. 2008. Fear conditioning and extinction differentially modify the intrinsic excitability of infralimbic neurons. J. Neurosci. 28:4028-4036.

113.Izhikevich, E.M., and Edelman, G.M. 2008. Largescale model of mammalian thalamocortical systems. Proc. Natl. Acad. Sci. U. S. A. 105:3593-3598.

114.Palomero-Gallagher, N., Vogt, B.A., Schleicher, A., Mayberg, H.S., and Zilles, K. 2008. Receptor architecture of human cingulate cortex: evaluation of the four-region neurobiological model. Hum. Brain Mapp. Online publication ahead of print. doi:10.1002/hbm.20667.

115.Pape, H.C., Narayanan, R.T., Smid, J., Stork, O., and Seidenbecher, T. 2005. Theta activity in neurons and networks of the amygdala related to long-term fear memory. Hippocampus. 15:874-880.

116. Fried, I., et al. 1999. Cerebral microdialysis combined with single-neuron and electroencephalographic recording in neurosurgical patients. Technical note. J. Neurosurg. 91:697-705.
117. McCracken, C.B., and Grace, A.A. 2007. Highfrequency deep brain stimulation of the nucleus accumbens region suppresses neuronal activity and selectively modulates afferent drive in rat orbitofrontal cortex in vivo. J. Neurosci. 27:12601-12610.

118.Belujon, P., and Grace, A.A. 2008. Critical role of the prefrontal cortex in the regulation of hippocampus-accumbens information flow. J. Neurosci. 28:9797-9805.

119.Zhang, F., Aravanis, A.M., Adamantidis, A., de Lecea, L., and Deisseroth, K. 2007. Circuit-breakers: optical technologies for probing neural signals and systems. Nat. Rev. Neurosci. 8:577-581.

120.Benabid, A.L., Pollak, P., Louveau, A., Henry, S., and de Rougemont, J. 1987. Combined (thalamotomy and stimulation) stereotactic surgery of the VIM thalamic nucleus for bilateral Parkinson disease. Appl. Neurophysiol. 50:344-346.

121.DeLong, M.R., and Wichmann, T. 2007. Circuits and circuit disorders of the basal ganglia. Arch. Neurol. 64:20-24.

122.Li, S., Arbuthnott, G.W., Jutras, M.J., Goldberg, J.A., and Jaeger, D. 2007. Resonant antidromic cortical circuit activation as a consequence of highfrequency subthalamic deep-brain stimulation. J. Neurophysiol. 98:3525-3537.

123. Trost, M., et al. 2006. Network modulation by the subthalamic nucleus in the treatment of Parkinson's disease. Neuroimage. 31:301-307.

124. Poston, K.L., and Eidelberg, D. 2008. Network biomarkers for the diagnosis and treatment of movement disorders. Neurobiol. Dis. Online publication ahead of print. doi:10.1016/j.nbd.2008.09.026. 